\title{
Clean versus sterile technique for common joint injections: a review from the physiatry perspective
}

\author{
Jennifer Baima $\cdot$ Zacharia Isaac
}

Published online: 17 November 2007

(C) Humana Press 2007

\begin{abstract}
Preparation of the skin prior to joint injection varies widely among disciplines and across regional borders. This is likely due to the paucity of literature on the most effective and efficient methods of preparation. There is no standard definition of clean technique prior to joint injection. Review of the available literature suggests that alcohol is effective preparation for the skin prior to most procedures. Surveys of current clinical practice demonstrate that the use of gloves may be favored, but no conclusions can be drawn in regards to whether sterile gloves are required. Clean technique should be defined as use of non-sterile gloves and agents such as alcohol or soap prior to injection. Significant cost savings may be achieved with the consistent use of clean technique for preparation of the skin prior to joint injection. Further study should address the incidence of iatrogenic bacterial arthritis following clean technique versus sterile technique for joint injection.
\end{abstract}

Keywords Intra-articular injection - Sterile technique . Clean technique $\cdot$ Joint infection

\section{Introduction}

Preparation of the skin prior to joint injection varies widely among disciplines and across regional borders. This is likely due to the paucity of literature on the most effective and efficient methods of preparation. Ideally, the most effective method of skin preparation would be that which precludes infection, a possibly preventable sequelae of intra-articular injection. The most efficient method would also be that which

J. Baima $(\varangle) \cdot$ Z. Isaac

Brigham and Women's Hospital, Boston, MA, USA

e-mail: jbaima1@partners.org demonstrates the lowest cost to the health care system. Sterile technique for operative procedures is known to carry a greater cost [1]. The efficacy and efficiency of sterile versus clean technique for joint injections has not been studied.

According to Taber's medical dictionary, aseptic or sterile technique is defined as "a method used in surgery to prevent contamination of the wound and operative site. All instruments used are sterilized, and physicians and nurses wear caps, masks, shoe coverings, sterile gowns, and gloves." [2] As most joint injections are done in ambulatory clinics, sterile technique often refers to the use of sterile gloves and drape for the procedure. Instruments are opened on to the "sterile field" and touched by the operator only prior to injection. As it is nearly impossible to replicate sterile procedures outside of the operating room, sterile technique for joint injections will be defined, for the purpose of the current discussion, as involving the use of sterile gloves and povidone-iodine or chlorhexidine solution prior to injection.

There is no known single definition of clean technique. In the wound care literature, clean technique has been defined as non-sterile technique involving "meticulous handwashing, maintaining a clean environment by preparing a clean field, using clean gloves and sterile instruments, and preventing direct contamination of materials and supplies." [3] As clean technique is the bare minimum required through Standard and Universal Precautions recommended by OSHA [4] at any ambulatory center in the United States, it will be defined for the purposes of this article as the use of non-sterile gloves and agents such as alcohol or soap prior to injection.

\section{Skin preparation agents}

One article has addressed the two different methods of skin preparation prior to joint injection. Cawley and Morris [5] 
compared the use of chlorhexidine skin preparation to isopropyl alcohol. There was no mention of the type of gloves used. The endpoint was the bacterial culture of the needles used for injection. This may not correlate with the organisms introduced into the joint. There was a lower incidence of culture-positive needles with the chlorhexidine skin preparation, but this was not statistically significant [5]. The organisms that grew out from the culture of the needles were not those commonly found in bacterial joint infections [6]. Although it is helpful to learn what organisms could potentially be isolated from culture of needles after different skin preparation techniques, the clinical relevance of this study is unknown.

Little is known about the efficacy of skin preparation agents. Two studies have addressed the current utilization of skin preparation with povidine-iodine or chlorhexidine versus alcohol prior to intra-articular injection in clinical practice. When 200 rheumatologists in the United Kingdom were asked their preferred agent, the list included mediswab (70\% isopropyl alcohol), hibitane (chlorhexidine), iodine, and ether in decreasing preference. About $57.5 \%$ were currently using alcohol wipes [7]. A later survey included 250 clinicians in the United Kingdom. More than half of these rheumatologists, orthopedists, and general practitioners favored alcohol swabs to povidoneiodine or chlorhexidine [8]. Both of these studies had nearly identical response rates of $76 \%[7,8]$. This demonstrates a subtle clinical trend toward alcohol swabbing prior to joint injection, which would fall under the above clean technique definition.

More extensive work has been done on skin preparation prior to surgical procedures. Most of this work has focused on comparison of different antiseptic agents [9]. As it is standard practice to prep with an antiseptic solution prior to surgical procedures in the operating room, comparison of chlorhexidine or povidine-iodine to alcohol or saline has not been performed in this country. Two studies have undertaken a comparison of these agents outside the United States. Kalantar-Hormosi and Davami report a comparison of chlorhexidine or povidone-iodine to soap and water cleansing with saline rinse in outpatient plastic surgical procedures. They reported on 905 cases in each group for minor skin procedures performed in the operating room with sterile technique [10]. The results of this study are concerning as there were no known cases of wound infection. Although it would be impossible to obtain the appropriate number of patients needed to validate this study, 1,800 patients is a large sample relative to the literature on skin preparation (Table 1).

Povidone-iodine skin preparation has been compared to market soap and methylated spirit (denatured ethanol) in preventing post-operative wound infections in patients in Nigeria after elective hernia repair [11]. Two hundred patients were randomized to skin preparation with either of the above agents. There was no significant difference between the two groups in terms of post-operative wound infection rate. There was no mention of the type of gloves used in this procedure, but sterile technique was the goal. The overall infection rate was $5.5 \%$, which is comparable to other rates in the area and likely was influenced by the limited availability of clean running water and electricity. If regular soap and alcohol is effective and efficient preparation prior to hernia repair, it may be all that is required for preparation prior to joint injection.

\section{Sterile gloves}

In the Cawley and Morris study [5], there is no mention of the type of gloves used. The chlorhexidine skin prep was applied with "full aseptic technique" which would include sterile gloves, but it was unclear if sterile gloves were in fact used with the alcohol skin preparation group. To date, no published study available by standard search methods has evaluated the use of sterile versus non-sterile gloves or no gloves prior to intra-articular injection. Yood conducted a survey of 23 rheumatologists to further elucidate current practice regarding the use of gloves with this procedure. $\mathrm{He}$ found that $50 \%$ of rheumatologists surveyed used gloves for "needle procedures of the joints, bursae, and tendons." Of this group, $25 \%$ consistently used sterile gloves [12]. Although this is a small sample of a cluster of rheumatologists in central Massachusetts, it demonstrates that perception of clean technique may not include use of any gloves at all.

A larger survey of 200 rheumatologists addressed the use of any gloves at all prior to intra-articular injection. Less than $10 \%$ of respondents utilized "surgical gloves." [7]. There was no clarification of the term "surgical gloves" in regards to sterility. A more recent survey of 50
Table 1 Literature about skin preparation

\begin{tabular}{llll}
\hline Authors & $\begin{array}{l}\text { Number of patients } \\
\text { excluding controls }\end{array}$ & $\begin{array}{l}\text { Type of } \\
\text { procedure }\end{array}$ & $\begin{array}{l}\text { Favors use } \\
\text { of alcohol swabs } \\
\text { over povidine-iodine }\end{array}$ \\
\hline Cawley and Morris [5] & 64 & Joint injection & Yes \\
Meier et al. [11] & 200 & Hernia repair & Yes \\
Kalantar-Hormosi and Davami [10] & 905 & Plastic surgery & No (soap then saline wash only) \\
\hline
\end{tabular}


general practitioners, 100 orthopedic surgeons, and 100 rheumatologists addressed the use of sterile gloves prior to steroid injection into the knee in the United Kingdom. Nearly $47 \%$ used either sterile or non-sterile gloves during the injection. About $32.5 \%$ routinely used sterile gloves for the procedure. The cost quoted in this article for sterile gloves was greater than four times the cost of non-sterile gloves [8]. According to the available literature, gloves are currently used about $50 \%$ of time. It is not surprising that there is no dominant clinical trend given the lack of data regarding the use of gloves in prevention of intra-articular infection (Table 2).

\section{Sterile versus clean technique}

Although no study in the United States has addressed infection after skin preparation with alcohol or soap prior to surgery, there has been evaluation of clean versus sterile surgical prep kits. The two skin preparation kits consisted of the same ingredients. One group of kits was assembled by hospital personnel using clean technique, and one group was from a manufacturer of sterile kits. Sixty patients were randomized to skin preparation with these kits. There was no significant difference between the two kits in terms of bacterial growth from cultured skin 10 min post-skin prep and at wound closure. The estimated savings in favor of using the clean surgical prep kit instead of the sterile surgical prep kit was $\$ 42,000$ annually at the hospital where the study was performed [1].

\section{Current practice}

Examining the perception of possible risk of joint injections may help us understand the marked variability in current use of sterile versus clean technique. Pal and Morris questioned 40 rheumatologists in the United Kingdom as to their recollection of occurrence of post-injection infection. Nearly $70 \%$ did not recall any cases of post-injection bacterial infection [13]. In another study involving 250 practitioners who perform joint injections, under $13 \%$ had encountered septic arthritis of the knee [8]. A larger study

Table 2 Literature about utilization of gloves

\begin{tabular}{llll}
\hline Authors & $\begin{array}{l}\text { Numbers } \\
\text { of surveys } \\
\text { sent }\end{array}$ & $\begin{array}{l}\text { Use of any } \\
\text { gloves (\%) }\end{array}$ & $\begin{array}{l}\text { Use of sterile } \\
\text { gloves (\%) }\end{array}$ \\
\hline Yood [12] & 23 & 50 & 25 \\
Haslock et al. [8] & 200 & & 9.8 \\
Charalambous et al. [7] & 250 & 46.6 & 32.5 \\
\hline
\end{tabular}

of perceived risks after intra-articular steroid injection by orthopedists involved 853 completed questionnaires. The perceived risk of infection was 1 in 1,000 in nearly $50 \%$ of surgeons polled and 1 in 10,000 in one third of the sample [14].

The prevailing theme among these surveys is the perception that intra-articular infection is not common. The actual reported incidence is unknown, but is thought to vary from 1: 3,000 to $1: 50,000$ [8]. If data is extrapolated from the above survey of rheumatologists [13], this would be 4.6 infections per 100,000 injections. It does appear that the perceived risk may be lower than the actual incidence. Although often catastrophic, the actual occurrence of these iatrogenic infections is so rare that a lower perceived risk than actual risk may not be clinically significant. There is also the question as to whether the etiology of the above injections was truly due to the introduction of skin flora through needling, as other more common mechanisms of bacterial arthritis have been described, such as hematogenous seeding [15].

\section{Discussion}

After observing attending rheumatologists, orthopedists, and physiatrists perform joint injections throughout residency, we have seen variations of the above-described sterile and clean techniques. This is likely due to the limited literature available. The swabs available in the abovementioned clinics are of two varieties: $70 \%$ isopropyl alcohol and $10 \%$ povidone-iodine. Neither agent, manufactured by Medline [16], mentions drying time in the box instructions. According to the surgical literature, povidoneiodine has peak bactericidal action when allowed to air dry for $20 \mathrm{~min}$ after application [17]. Although we have observed use of povidine-iodine prior to injection on multiple occasions, we have never seen the operator pause more than a few seconds after application before injection. It is possible that we are not taking advantage of the bactericidal action of povidine-iodine.

Another important issue is the use of sterile kits. In the surgical literature, there is no known benefit to the use of sterile preparation kits over clean preparation kits in preventing wound infection. A retrospective study in France estimated one episode of sepsis after local corticosteroid injection per 77,300 procedures. Nine out of fifteen of the cases reviewed occurred when the steroid was not packaged in a sterile syringe [18]. To date, no studies have evaluated a pre-packaged joint injection kit to minimize transfer of office flora to injected ingredients.

Of note, other common office procedures are performed with the use of alcohol only. For example, both electromyography needle placement and trigger point injection 
involve penetrating the skin and underlying muscle with a needle. Common practice seems to be skin preparation with an alcohol swab only. In a PubMed search, we were able to find only one case report of soft tissue infection after electromyography. This was due to mycobacterium fortuitum thought to be living on reusable needle electrodes [19]. This is not a common cause of bacterial arthritis. Although intra-muscular injections are likely to be less invasive than intra-articular, it is interesting that there is no agreed upon recommendation for these procedures.

Joint aspiration is commonly performed by rheumatologists. Kelly's Textbook of Rheumatology [20] cites the Cawley and Morris [5] article in favor of single alcohol swab, but then recommends that "the area should be carefully cleaned with one or two layers of iodine followed by alcohol." We have often seen this performed in the office. It is possible that since these two agents have different mechanisms of action, their use could have a synergistic effect. A recent review article supports the use of "combination formulations" of these two agents to achieve broader coverage [21].

Alcohol pads cost approximately 3 cents per individual pad and povidone-iodine costs 13 cents per pad on one medical supply website [22]. Sterile gloves are considerably more expensive than non-sterile gloves. If purchased in bulk, a consumer website lists sterile gloves at $\$ 170.00$ per 100 pair and non-sterile gloves at under $\$ 3.00$ per 100 pair [23]. Prices vary with the market, but sterile gloves appear to be over 50 times more expensive. Review of the available literature suggests that alcohol is effective preparation for the skin. Review of surveys of current clinical practice suggests the use of gloves may be favored, but no conclusions can be drawn in regards to what type of gloves. Significant cost savings may be achieved with the consistent use of clean technique for preparation of the skin prior to joint injection. Further study should address the incidence of iatrogenic bacterial arthritis following clean technique for joint injection.

\section{Summary recommendation}

Based on the current existing literature, use of clean technique prior to joint injection seems to be comparable to sterile technique in safety and can be performed at lower cost, clean technique being defined as use of handwashing, non-sterile gloves, and alcohol swab prior to injection.

\section{References}

1. Pearce BA, Miller LH, Martin MA, Roush DL. Efficacy of clean v sterile surgical prep kits. AORN J 1997;66(3):464-70.
2. Venes D. Taber's cyclopedic medical dictionary. Philadelphia: FA Davis Company; 2005.

3. Wooten MK, Hawkins K. Clean versus sterile: management of chronic wounds. WOCN Position Statement. January 2005.

4. Occupational and Safety Health Administration Regulations: Occupational Safety and Health Standards, Toxic and Hazardous Substances, 1910.1030. www.osha.gov. Accessed 1 Feb 2007.

5. Cawley PJ, Morris IM. A study to compare the efficacy of two methods of skin preparation prior to joint injection. Br J Rheumatol 1992;31:847-8.

6. Ryan MJ, Kavanagh R, Wall PG, Hazleman BL. Bacterial joint infections in England and Wales: analysis of bacterial isolates over a four year period. Br J Rheumatol 1997;36:370-3.

7. Charalambous CP, Tryfonidis M, Sadiq S, Hirst P, Paul A. Septic arthritis following intra-articular steroid injection of the knee - a survey of current practice regarding antiseptic technique used during intra-articular steroid injection of the knee. Clin Rheumatol 2003;22:386-90.

8. Haslock I, Macfarlane D, Speed C. Intra-articular and soft tissue injections: a survey of current practice. $\mathrm{Br} \mathrm{J}$ Rheumatol 1995;34:449-52.

9. Ritter MA, French ML, Eitzen HE, Goie TJ. The antimicrobial effectiveness of operative-site preoperative agents: a microbiological and clinical study. JBJS 1980;62:826-8.

10. Kalantar-Hormozi AJ, Davami B. No need for preoperative antiseptics in elective outpatient plastic surgical operations: a prospective study. Plast Reconstr Surg 2005;116(2):529-31.

11. Meier DE, Nkor SK, Aasa D, OlaOlorun DA, Tarpley JL. Prospective randomized comparison of two preoperative skin preparation techniques in a developing world country. World $\mathbf{J}$ Surg 2001;25:441-3.

12. Yood RA. Use of gloves for rheumatology procedures. Arthritis Rheum 1993;36(4):575.

13. Pal B, Morris J. Perceived risks of joint injection following intraarticular corticosteroid injections: a survey of rheumatologists. Clin Rheumatol 1999;18:264-5.

14. Farooq MA, Devitt AT. Perceived efficacy and risks of infection following intra-articular injections: a survey of orthopedic surgeons. Ir J Med Sci 2005;174(1):26-32.

15. Kaandorp CJE, Dinant HJ, van de Laar MAFJ, Moens HJB, Prins APA, Dijkmans BAC. Incidence and sources of native and prosthetic joint infection: a community based prospective survey. Ann Rheum Dis 1997;56:470-5.

16. Package insert, available at www.medline.com, Medline Industries, Inc. Mundelein, IL 60060 USA. Accessed 1 Feb 2007.

17. Workman ML. Comparison of blot-drying versus air-drying of povidone-iodine cleansed skin. Appl Nurs Res 1995;8(1):15-7.

18. Seror P, Pluvinage P, d'Andre FL, Benamou P, Attuil G. Frequency of sepsis after local corticosteroid injection (an inquiry on 1160000 injections in rheumatological private practice in France). Rheumatology (Oxford) 1999;38(12):1272-4.

19. Nolan CM, Hashisaki PA, Dundas DF. An outbreak of soft-tissue infections due to Mycobacterium fortuitum associated with electromyography. J Infect Dis 1991;163(5):1150-3.

20. Harris ED, et al. Kelley's textbook of rheumatology. Philadelphia: Elsevier Science; 2005. p. 698.

21. Art G. Combination of povidone-iodine and alcohol formulations more effective, more convenient versus formulations containing either iodine or alcohol alone: a review of the literature. J Infus Nurs 2005;28(5):314-20.

22. www.brucemedical.comwww.brucemedical.com, Bruce Medical Supply, 411 Waverly Oaks Road, Suite 154 Waltham, MA 02452. Accessed 1 Feb 2007.

23. Online price catalogue available at http://www.glovesaver.com. Accessed 1 Feb 2007. 